\title{
Learn to See: A Microwave-based Object Recognition System Using Learning Techniques
}

\author{
Viktor Erdélyi \\ viktor@ist.osaka-u.ac.jp \\ Osaka University \\ Osaka, Japan \\ Hirozumi Yamaguchi \\ h-yamagu@ist.osaka-u.ac.jp \\ Osaka University \\ Osaka, Japan
}

\author{
Hamada Rizk \\ hamada_rizk@ist.osaka-u.ac.jp \\ Osaka University, Osaka, Japan \\ Tanta University, Tanta, Egypt \\ Teruo Higashino \\ higashino@ist.osaka-u.ac.jp \\ Osaka University \\ Osaka, Japan
}

\begin{abstract}
The capability to recognize nearby objects automatically has numerous applications including asset tracking, lifestyle analysis, and navigation assistance for blind people. In recent years, several approaches were proposed, but they are either limited to electric objects or objects instrumented with tags, which cannot scale. There are also acoustic or vision-based techniques for recognizing uninstrumented objects, but they may have privacy issues.

In this paper, we present a microwave-based object detection and recognition approach. Specifically, the proposed system leverages Universal Software Radio Peripherals (USRPs) to transmit microwave signals through the target object and capture them on the opposite side. To reduce the privacy impact, we use a single antenna for receiving a single-pixel "image". Then, a Random Forest classifier learns the characteristics of the received signals altered by a given object, enabling object recognition.

Using a wide range of microwave frequencies, we evaluated the proposed system's capability to detect and differentiate between four different objects of different materials. The evaluation results show that, using only a $10 \mathrm{~ms}$ signal, the system can correctly detect the presence of the object $98.7 \%$ of the time. The system can also differentiate between different objects $92 \%$ of the time.
\end{abstract}

\section{CCS CONCEPTS}

-Human-centered computing $\rightarrow$ Ubiquitous and mobile computing systems and tools; • Networks $\rightarrow$ Cognitive radios; • Hardware $\rightarrow$ Digital signal processing; • Computing methodologies $\rightarrow$ Supervised learning by classification.

\section{KEYWORDS}

uninstrumented object recognition, microwave sensing, softwaredefined radio, machine learning

Permission to make digital or hard copies of part or all of this work for personal or classroom use is granted without fee provided that copies are not made or distributed for profit or commercial advantage and that copies bear this notice and the full citation on the first page. Copyrights for third-party components of this work must be honored. For all other uses, contact the owner/author(s).

ICDCN '21 Adjunct, fanuary 5-8, 2021, Nara, Japan

(C) 2021 Copyright held by the owner/author(s).

ACM ISBN 978-1-4503-8184-0/21/01.

https://doi.org/10.1145/3427477.3429459

\section{ACM Reference Format:}

Viktor Erdélyi, Hamada Rizk, Hirozumi Yamaguchi, and Teruo Higashino. 2021. Learn to See: A Microwave-based Object Recognition System Using Learning Techniques. In Adjunct Proceedings of the 2021 International Conference on Distributed Computing and Networking (ICDCN '21 Adjunct), January 5-8, 2021, Nara, Japan. ACM, New York, NY, USA, 6 pages. https: //doi.org/10.1145/3427477.3429459

\section{INTRODUCTION}

The capability to recognize nearby objects automatically has many applications in context sensing. For example, in industrial environments, various assets can be tracked effectively and without human intervention. Additionally, by sensing a person's interactions with their environment and the objects contained in that environment, fine-grained information can be obtained about their lifestyle, enabling novel applications based on the analysis of the interaction data. Yet another application is to assist navigation for visually impaired people: information about their surroundings helps them find their belongings and navigate around obstacles.

By object recognition, we refer to inferring what kind of object we are dealing with. It is different from object detection, which produces a binary output (is there an object or not), and also different from object identification, which aims to identify which object we are dealing with, even among many instances of the same type.

In recent years, numerous approaches have been proposed, but many of them either rely on smart objects that can communicate according to a well-defined communication protocol, or they need the objects to be instrumented with tags, e.g. Radio-Frequency Identification (RFID) [21] tags. As the number of objects increase, the use of smart objects raises concerns about battery replacement, making maintenance difficult. RFID tags, on the other hand, incur a deployment cost by having to attach tags to each object of interest, and also has an environmental impact when disposing of tags. In order to alleviate the environmental concerns, FerroTag [11] proposes the use of environment-friendly ferrofluidic ink. However, the object shape might not allow for tag attachment. Thus, in order to support a diverse set of objects in a maintenance-free way, it is desirable to support plain and simple objects.

One potential approach to recognizing objects is the use of electromagnetic waves. As confirmed in spectroscopy-related literature [7], objects (depending on their material even plain objects) have a unique response to incoming electromagnetic waves. For instance, 
the USGS Spectral Library [7] contains spectral reflectance information for various materials, covering wavelengths from the ultraviolet to the far infrared $(0.2$ to $200 \mu \mathrm{m})$ and showing significant variance across materials.

Another relevant region of the electromagnetic spectrum is $m i-$ crowaves. Due to the widespread availability of software-defined radios, microwave signals are relatively easy to work with, and are safer than some of their higher-frequency counterparts such as UV or X-rays.

Additionally, when energy is transmitted from a certain microwave source towards an object, depending on the molecular structure of the object and the frequency of the wave, the object may have a unique response [8]. Motivated by this property of materials and by the recent advancement of machine learning approaches, we attempt to answer the following question: is it possible to recognize different types of uninstrumented objects using microwave signals?

In this work, we propose a microwave-based object detection and recognition system that achieves high accuracy with uninstrumented objects ${ }^{1}$. Specifically, the system transmits a white noise signal in a given frequency band using a pair of antennas, each connected to a USRP radio $[18,19]$. Using only a single pair of antennas means that the (spatial) resolution of the final microwave "image" is only $1 \mathrm{x} 1$ pixel, which reduces the privacy impact of our technique. The system lets the transmitted signal pass through the target object, and then records the altered signal on the opposite side of the object. The proposed system incorporates different modules to extract discriminative features from the received signals, which facilitates the training of a Random Forest classifier for object detection and recognition purposes.

Our contributions are as follows:

- We present the design and implementation of a system to recognize objects based on their differences in interactions with microwave signals.

- In order to prove the feasibility of our approach, we present a preliminary evaluation using four objects, which shows that our approach can achieve $98.7 \%$ object detection accuracy as well as $92 \%$ object recognition accuracy. These results confirm that microwave signals can be considered a promising modality for enabling the next generation of object detection and recognition.

The rest of the paper is organized as follows: We present our system design in Section 2 and discuss our system implementation in 3. We evaluate our design in Section 4, discuss the related work in Section 5, and we conclude in Section 6.

\section{SYSTEM DESIGN}

In this section, we present our design. First, we discuss the requirements, then we explain various design decisions we had to make, such as what frequency, what signal type, what sensing resolution and what sensing approach to use.

To build a practical object recognition system, several requirements must be satisfied: the system should be easy to deploy and maintain on a large scale at a low cost, and thus it should require as little infrastructure as possible. As for the objects, in order to

\footnotetext{
${ }^{1}$ no tags are required
}

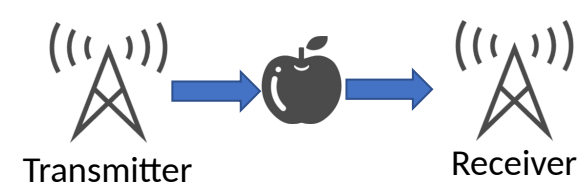

Figure 1: Passthrough method. The transmitted signal passes through the object before it reaches the receiver.

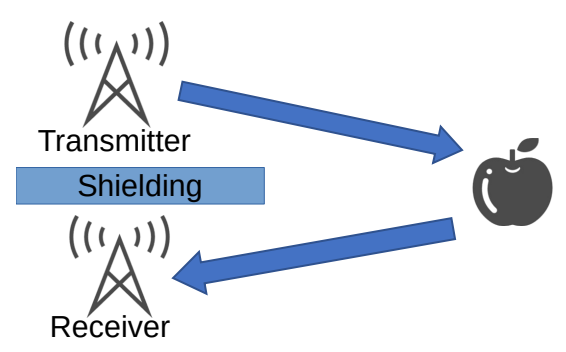

Figure 2: Reflection method. The transmitted signal is reflected off of the object before it reaches the receiver. Shielding is added to prevent the signal from propagating directly to the receiver without touching the object.

eliminate power-related maintenance costs and the potential environmental impact of batteries and tags, it is desirable to design a method that requires no instrumentation. Note that, since we cannot assume any pre-programmed logic inside the objects, this approach is the most challenging one. As for the sensing equipment, since the number of sensors is expected to be much smaller than the number of objects, the requirements are not as strict. In this work, we adopt an approach that involves connecting two software-defined radio units to a computer via USB, and we leave the construction of a more compact, portable prototype as future work.

\subsection{Choosing a frequency band}

A fundamental property of waves is their frequency (and the corresponding wavelength). In fact, the most fundamental difference among all electromagnetic waves is their frequency [14]. The rest of their properties, such as how they interact with physical matter, are a consequence of their frequency. Since some objects may react very similarly to incoming energy at specific frequencies, not all frequencies may be discriminative enough for recognizing objects. Therefore, it is important to explore a wide range of frequencies and choose one or more based on the objects of interest.

In this paper, we set out to investigate the response of objects to microwave frequencies in particular, and we evaluate the feasibility of using such microwaves for recognizing objects. We selected the widest range of frequencies that can be used with the combination of transmitters, receivers and antennas we have. For our experiments, we used the 0.85 to $6 \mathrm{GHz}$ frequency band. 


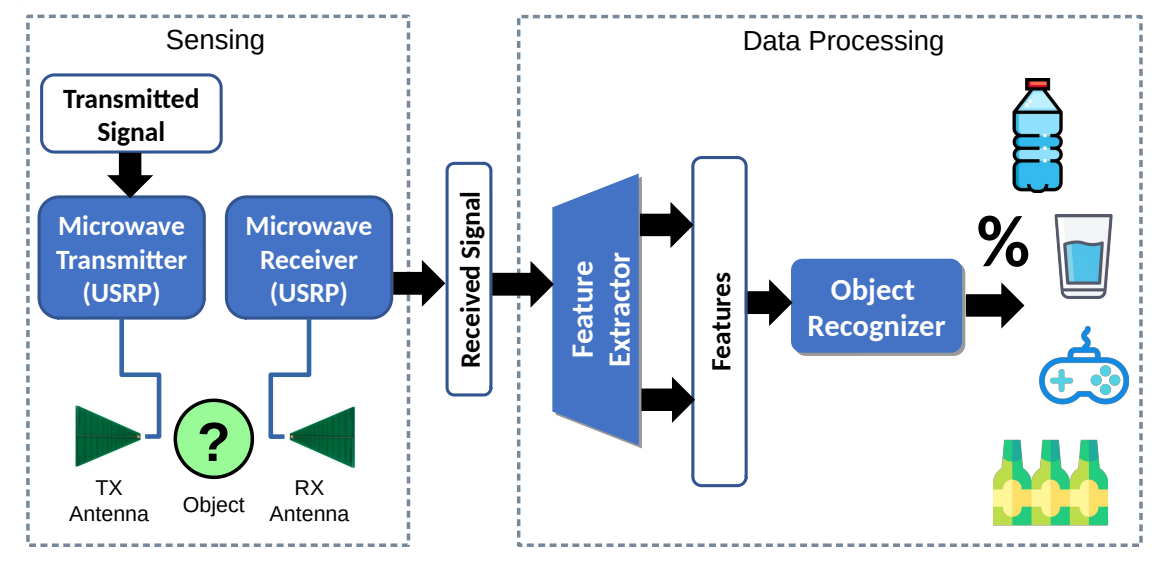

Figure 3: System Architecture

\subsection{Choosing a signal for transmission}

When transmitting a signal, we are not trying to perform digital data transmission (as in encoded/modulated bits) over the air. We are only interested in statistical information about the signal. Therefore, in order to have (nominally) equal power for each transmitted frequency, we chose a white noise signal because of its flat spectrum.

\subsection{Spatial resolution vs. spectral resolution}

In the visible light frequency range (and sometimes also in the $\mathrm{UV} /$ infrared range), sensing is commonly done by cameras [16]. Cameras typically produce a $2 \mathrm{D}$ image of a specific resolution, allowing us to obtain information about the shape of objects. However, a high-resolution image (even in the UV/infrared range) may be perceived as highly private data, raising privacy concerns and calling for advanced privacy-preserving processing techniques [2].

In order to alleviate the privacy concerns, an alternative approach is to lower the resolution intentionally, in an extreme case down to only $1 \mathrm{x} 1$ pixel, and, in order to compensate for the lost information, increase the spectral resolution (the number of frequencies being sensed) instead. For example, instead of obtaining an RGB image (only 3 channels), we could obtain a reading that includes a perfrequency intensity value for a larger set of frequencies. In this paper, we use this latter approach.

\subsection{Choosing a sensing approach}

The energy transmitted towards an object will result in various types of interactions depending on the position of the transmitter and receiver relative to the object [15]. (Note that any emission by the object itself will be added to the received signal.) If we position the object between a transmitter and a receiver, the signal (or some components thereof) will pass through the object (Figure 1). On the other hand, if we position the transmitter and receiver on the same side of the object, we can observe the signal components reflected by the object. In order to avoid self-interference, we may need to place an RF shield between the transmitter and receiver, as shown in Figure 2. Due to its simplicity (no shielding required), we use the passthrough approach.
The next decision to make is where to get the energy from. When there is an appropriate external energy source (in terms of intensity and frequency), a passive sensor can be used. For instance, human vision uses reflections of external energy (from the Sun or from artificial lighting) in a narrow frequency range. If there is no such energy source, an additional energy source is needed. This approach is called "active sensing". Since the background microwave radiation is either weak (such as the cosmic microwave background) or unpredictable (such as Wi-Fi traffic), we decided to use the active sensing approach.

\section{IMPLEMENTATION}

In this section, we start by an overview of the proposed system. Then, we give an explanation of each of its components.

\subsection{System overview}

Figure 3 shows the system architecture. First, the Sensing module obtains and records the EM signal in a passthrough configuration. Then, the Data Processing module processed the recorded data. Within this module, the Feature Extractor module first extracts salient features for object recognition. The extracted features are then forwarded to the Object Recognizer module, which outputs the predicted object type.

The Object Recognizer module works in two stages: an offline training stage and an online recognition stage. In the offline stage, we construct and train an optimal object recognition model using the features and object labels as input. Additionally, we identify the frequency that maximizes the accuracy across all objects in the training data. The output of this offline stage is a trained recognition model. In the online phase, we classify unknown objects by feeding the extracted feature data directly into the trained recognition model and identifying the object class that is most correlated to the input features.

\subsection{Sensing module}

This module consists of a transmitter and a receiver. It operates as follows. First, we start the receiver. When the first batch of samples 
come in, the receiver sends a start signal to the transmitter. The transmitter then upconverts a predefined white noise signal to the desired center frequency and transmits it from the TX antenna. When the whole signal has been transmitted, the transmitter stops and sends a stop signal to the receiver. The receiver receives the (modified) signal on the RX antenna, downconverts it, and then records the resulting (baseband) signal. (By doing up- and downconversion, the host computer can operate at a lower, more manageable sampling rate.) This module is implemented in Kotlin/JVM using a JVM binding of the UHD (USRP Hardware Driver) library for communicating with the USRPs.

\subsection{Feature Extractor module}

The goal of this module is to extract features from the received baseband radio signal using a windowing approach. For each (nonoverlapping) window of $10 \mathrm{~ms}$ length, we compute a total of 12 statistical features including: Median, Inter-Quartile range, Skewness, Max Spectral Entropy, Zero Crossings, Max Autocorrelation, Variance, Standard Deviation, Mean, Root-Mean-Square (RMS), Minimum, Maximum. This module is implemented in MATLAB.

\subsection{Object Recognizer module}

In this section, we describe how we build a classification model that, using the time-domain features of the received signal, can precisely differentiate between different objects. We train different classifiers including logistic regression classifier, one-vs-all Support Vector Machine classifier, K-Nearest Neighbors classifier, and Decision Tree. We found that each individual classifier achieves a non-satisfactory performance due to its limited learning ability for the underlying process. Therefore, we decided to use a Random Forest Classifier. The Random Forest contains a large number of decision trees. Each of them outputs a class prediction, and the class that has the most votes becomes our model's prediction.

The motivation for using a Random Forest classifier is its ability to train a large number of relatively uncorrelated models (trees) operating as a ensemble of predictors that are more accurate than individual ones. Additionally, Random Forest is a flexible, easyto-use supervised learning algorithm that produces, even without hyper-parameter tuning, a great result most of the time. It is also one of the most used algorithms because of its simplicity and diversity. The input to the classifier is the features obtained from the feature extraction module. The best performance has been obtained with 200 trees and the entropy as a split metric. Once the model has been trained, it can be used to recognize the object in question given the extracted radio-based features.

\section{EVALUATION}

In this section, we present our evaluation of the proposed system. First, we describe our experiment setup. Then, we present our experimental results. Finally, we outline some directions for future work.

\subsection{Experiment setup}

For our sensing setup, we used a USRP B205mini [18] SDR as the transmitter, and a USRP B210 [19] SDR as the receiver unit, and connected an LP0965 log-periodic antenna [17] to each of them.
(We used two USRP units in order to reduce self-interference.) We configured the transmitter to have a TX gain of 60 , and the receiver to have an RX gain of 76. We used 16-bit samples at a baseband sampling rate of $300 \mathrm{kHz}$.

In our experiments, we placed the transmitter and receiver antennas about $26.5 \mathrm{~cm}$ apart into a MICRONIX MY3720 Electromagnetic Anechoic Box [13], and placed the target object in between the transmitter and receiver antennas (passthrough configuration).

We used the following objects for our experiments:

- A 2 L PET bottle (full of water)

- One $2 \mathrm{~L}$ and two 0.9 L PET bottles (full of water) arranged on a line (so that the signal has to go through all 3 bottles)

- A $270 \mathrm{~mL}$ stainless steel drinking glass (empty)

- A PlayStation 4 DualShock wireless game controller (turned off)

For each object (and also without any object), we transmitted a pre-generated white noise signal at several frequencies ranging from $850 \mathrm{MHz}$ to $6 \mathrm{GHz}$ (in $100 \mathrm{MHz}$ steps). For each frequency and for each object, we collected around 340000-356000 baseband samples. Then, we split the data into time slots of 3000 samples (about $10 \mathrm{~ms}$ ) each, and computed features for each time slot.

\subsection{Experimental results}

In this section, we investigate the effect of changing the frequency on detection accuracy. Figure 4 shows the frequency at which the highest accuracy per object is obtained. The figure shows that the optimal frequency for detection of plastic bottles, stainless steel drinking glass and game controller are of $4 \mathrm{GHz}, 1.8 \mathrm{GHz}$ and $2.9 \mathrm{GHz}$, respectively. The best frequency is the one at which the received signals (after passing through the object) are the most unique compared to the set of remaining objects. The actual value of the best frequency is presumably due to the differences in the molecular structure of the object material (note that the two objects that consist mainly of water have a similar best frequency), but further investigation is needed. The best overall performance for classifying all objects, as shown in Figure 5, is achieved at $1.85 \mathrm{GHz}$. This result suggests that the received signals have discriminating patterns for different objects at this frequency.

\subsection{Future work}

In this section, we outline some possible future directions for our work.

- More frequencies: We plan to extend our work to use more frequency bands, potentially going beyond the microwave frequency range.

- Frequency-domain features: Instead of using only time-domain features, we intend to use frequency-domain (spectral) features as well.

- Portable prototype: We plan to build a portable prototype with adjustable antennas and low-power processing units, e.g. microcontrollers.

- Reflection method: In addition to the passthrough method, we plan to explore the reflection method as well. 


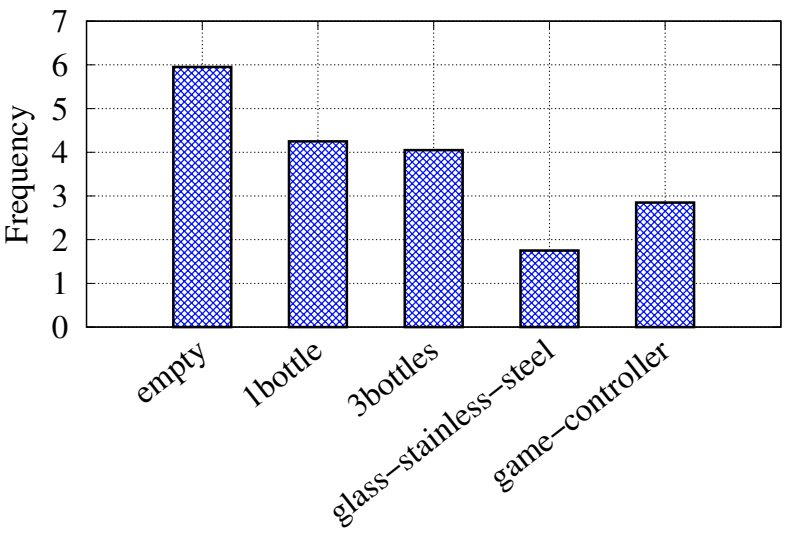

Object

Figure 4: Frequency for best object detection $(\mathrm{GHz})$

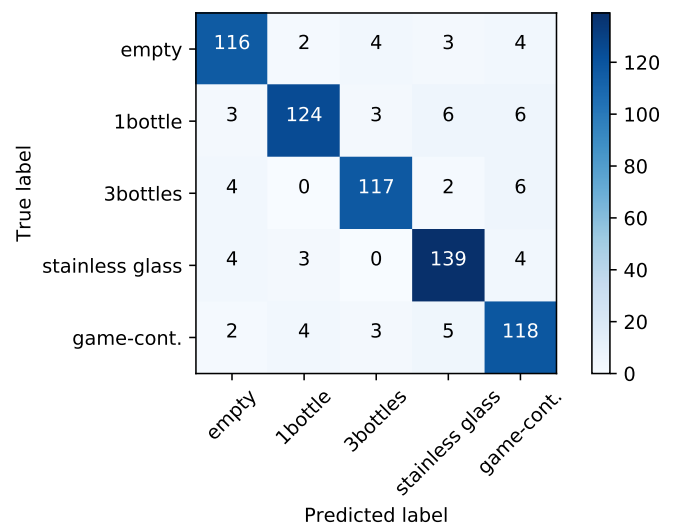

Figure 5: Confusion matrix

\section{RELATED WORK}

In recent years, several object recognition approaches have been explored. They can be broadly categorized into (1) recognizing active objects, which emit some form of wave, most commonly an EM wave, and (2) recognizing passive objects, which do not emit any wave. (They do emit EM radiation in the form of black-body radiation based on their temperature, but that may not fall into the desired frequency range, and temperature by itself may not be a discriminative feature.) In this section, we discuss these approaches in detail.

\subsection{Recognizing active objects}

The emissions of active objects can be captured using a softwaredefined radio (SDR). For example, EM-Sense [10], Deus EM Machina [22] and EM-ID [23] use a modified RTL-SDR [20] to record emissions in a subset of the frequency range 0 to $2 \mathrm{MHz}$.
EM-Sense uses the body antenna effect to recognize electrical devices on touch or on activation. The prototype includes a smartwatch, an SDR, a smartphone, and a laptop. Deus EM Machina provides additional contextual functionality by launching a contextspecific user interface on an IoT appliance. Their sensing hardware is more compact (antenna, microcontroller, and a smartphone), inexpensive, and all processing happens on the phone itself. Both systems classify objects using SVM. While this approach does not require us to transmit any signal or attach any tags to the objects, it does not support plain, non-electrical objects.

\subsection{Recognizing passive objects}

To recognize passive objects, one needs to send energy towards the object and observe the resulting interaction. For better performance, objects can be instrumented using tags that interact with the incoming energy in a recognizable way. Alternatively, recognition may be attempted without instrumentation. In this section, we consider these cases in detail.

5.2.1 Instrumented (tagged) objects. A typical approach is based on RFID. For example, Lakafosis et al. [9] and Bertoncini et al. [1] target anti-counterfeiting applications using instrumented objects (RFID tags).

An improvement over RFID is FerroTag [11], which provides an ultra-low cost, environment-friendly and battery-free tagging technique. FerroTag uses complex, 2 to $3 \mathrm{~cm}$ large circular patterns that can be printed on objects with ferrofluidic ink, which reflect millimeter-wavelength signals in an identifiable way. These reflections can be received from $60 \mathrm{~cm}$ distance even in non-line of sight conditions within around $20 \mathrm{~ms}$. FerroTag uses temporal and spectral features and a Random Forest classifier, and achieves over $99 \%$ accuracy in identifying tags.

However, these techniques require object instrumentation. In contrast, we focus on recognizing uninstrumented (plain, untagged) objects.

5.2.2 Uninstrumented objects. There are two main approaches towards recognizing uninstrumented objects. One approach works by creating a mechanical impact on the object and observing the resulting sound. The other approach is to transmit an electromagnetic wave towards the object, and observe how the object alters the signal.

An example of a sound-based approach is Knocker [5], which requires that a user knocks on the object with a smartphone. In addition to the sound generated by the impact, Knocker also records accelerometer and gyroscope data from the smartphone and then classifies the response using SVM. A similar system, Knock-Knock [12], can recognize 30 objects based on the sound of knocking on the object with a marker pen. Knock-Knock uses stacked denoising autoencoders to train a deep learning model using 120 knocks per object.

Some objects such as fragile or easily bendable ones, however, are not suitable for knocking. Additionally, the sound produced by knocking may disturb users. Finally, the need for mechanical contact limits the sensing range significantly, and may facilitate the transmission of infectious diseases. Thus, we use electromagnetic waves instead. 
Regarding the electromagnetic approaches, it is common to use the human-visible portion of the electromagnetic spectrum. Beyond standard computer vision methods, a unique approach is proposed by Kacorri et al. [6], where visually impaired people train a deep classification model with a few snapshots of objects of interest. However, in standard visible light-based approaches, the high image resolution makes the captured data privacy-sensitive.

An alternative option is microwave signals. Recent advances in microwave technology have enabled the construction of cameras $[3,4]$ that operate in the microwave range. Such cameras use an antenna array or the synthetic aperture radar technique to create images, and include microwave transmitter(s) that serve as a "camera flash". However, these cameras are still complex and bulky. Additionally, if the image is too detailed, it might be seen as sensitive data. Thus, we instead use a standard antenna to create a $1 \mathrm{x} 1$ pixel "camera" for microwave reception (and another antenna for "illuminating" the object).

\section{CONCLUSION}

We proposed a system to recognize objects based on differences in their interactions with microwave signals. The proposed system includes a Sensing Module, which transmits and captures microwave signals in a pass-through manner using a software-defined radio platform, and Data Processing Modules, which extract useful timedomain features and classify various objects using a Random Forest technique.

We evaluated the proposed system using a wide range of microwave frequencies and four different objects having different molecular structures, and we achieved a consistent object detection and recognition accuracy of $98.7 \%$ and $92 \%$, respectively. The system needs only a $10 \mathrm{~ms}$ signal to achieve this accuracy. Based on our results, we conclude that it is feasible to detect and recognize objects using microwave signals. We believe that the proposed system contributes a useful new modality to the sensing arsenal of future electromagnetic sensing systems.

\section{ACKNOWLEDGMENTS}

This work is supported in part by Grant-in-Aid for Scientific Research (S) (Grant Number 19H05665) from JSPS (Japan Society for the Promotion of Science).

\section{REFERENCES}

[1] Crystal Bertoncini, Kevin Rudd, Bryan Nousain, and Mark Hinders. 2011. Wavelet fingerprinting of radio-frequency identification (RFID) tags. IEEE Transactions on Industrial Electronics 59, 12 (2011), 4843-4850.

[2] University of Florida FOCUS Lab. 2020. Privacy Preserving Computational Cameras. Retrieved 2020-08-22 from http://focus.ece.ufl.edu/privacy_preserving computational_cameras/
[3] Mohammad Tayeb Ghasr, Mohamed A Abou-Khousa, Sergey Kharkovsky, Reza Zoughi, and David Pommerenke. 2011. Portable real-time microwave camera at $24 \mathrm{GHz}$. IEEE Transactions on Antennas and Propagation 60, 2 (2011), 1114-1125.

[4] Mohammad Tayeb Ghasr, Matthew J Horst, Matthew R Dvorsky, and Reza Zoughi. 2016. Wideband microwave camera for real-time 3-D imaging. IEEE Transactions on Antennas and Propagation 65, 1 (2016), 258-268.

[5] Taesik Gong, Hyunsung Cho, Bowon Lee, and Sung-Ju Lee. 2019. Knocker: Vibroacoustic-Based Object Recognition with Smartphones. Proceedings of IMWUT (Interactive, Mobile, Wearable and Ubiquitous Technologies) (2019). https: //doi.org/10.1145/3351240

[6] Hernisa Kacorri, Kris M. Kitani, Jeffrey P. Bigham, and Chieko Asakawa. 2017. People with Visual Impairment Training Personal Object Recognizers: Feasibility and Challenges. In Proceedings of CHI (Conference on Human Factors in Computing Systems). https://doi.org/10.1145/3025453.3025899

[7] R.F. Kokaly, R.N. Clark, G.A. Swayze, K.E. Livo, T.M. Hoefen, N.C. Pearson, R.A. Wise, W.M. Benzel, H.A. Lowers, R.L. Driscoll, and A.J. Klein. 2017. USGS Spectral Library Version 7: U.S. Geological Survey Data Series 1035. https://doi.org/10.3133/ ds1035

[8] Ivo Křivánek. 2008. Dielectric properties of materials at microwave frequencies. Acta Universitatis Agriculturae et Silviculturae Mendelianae Brunensis (2008). https://doi.org/10.11118/actaun200856050125

[9] Vasileios Lakafosis, Anya Traille, Hoseon Lee, Edward Gebara, Manos M Tentzeris, Gerald R DeJean, and Darko Kirovski. 2010. RF fingerprinting physical objects for anticounterfeiting applications. IEEE Transactions on Microwave Theory and Techniques 59, 2 (2010), 504-514.

[10] Gierad Laput, Chouchang Yang, Robert Xiao, Alanson Sample, and Chris Harrison. 2015. EM-Sense: Touch Recognition of Uninstrumented, Electrical and Electromechanical Objects. In Proceedings of the 28th Annual ACM Symposium on User Interface Software \& Technology. https://doi.org/10.1145/2807442.2807481

[11] Zhengxiong Li, Baicheng Chen, Zhuolin Yang, Huining Li, Chenhan Xu, Xingyu Chen, Kun Wang, and Wenyao Xu. 2019. FerroTag: A Paper-Based MmWaveScannable Tagging Infrastructure. In Proceedings of SenSys (Conference on Embedded Networked Sensor Systems). https://doi.org/10.1145/3356250.3360019

[12] Shan Luo, Leqi Zhu, Kaspar Althoefer, and Hongbin Liu. 2017. Knock-knock: acoustic object recognition by using stacked denoising autoencoders. Neurocomputing 267 (2017), 18-24.

[13] MICRONIX. [n.d.]. Hand-in type Electromagnetic Anechoic Box MY3720. Retrieved 2020-08-20 from http://www.micronix-jp.com/english/products/shieldbox/hand-in-type/my3720.html

[14] National Aeronautics and Space Administration (NASA). 2013. The Electromagnetic Spectrum. Retrieved 2020-08-22 from https://imagine.gsfc.nasa.gov/science/ toolbox/emspectrum1.html

[15] National Aeronautics and Space Administration, Science Mission Directorate. 2010. Wave Behaviors. Retrieved 2020-08-22 from https://science.nasa.gov/ems/ 03 behaviors

[16] Spencer's Camera \& Photo. 2020. Full Spectrum Converted Cameras. Retrieved 2020-08-22 from https://www.spencerscamera.com/full-spectrum-cameras.cfm

[17] Ettus Research. 2020. LP0965 Antenna. Retrieved 2020-08-22 from https://www. ettus.com/all-products/lp0965/

[18] Ettus Research. 2020. USRP B205mini-i. Retrieved 2020-08-22 from https://www. ettus.com/all-products/usrp-b205mini-i/

[19] Ettus Research. 2020. USRP B210. Retrieved 2020-08-22 from https://www.ettus. com/all-products/ub210-kit/

[20] RTL-SDR. 2020. RTL-SDR (RTL2832U) and software defined radio. Retrieved 2020-08-22 from https://www.rtl-sdr.com/

[21] U.S. Food and Drug Administration. 2018. Radio Frequency Identification. Retrieved 2020-08-22 from https:/www.fda.gov/radiation-emitting-products/ electromagnetic-compatibility-emc/radio-frequency-identification-rfid

[22] Robert Xiao, Gierad Laput, Yang Zhang, and Chris Harrison. 2017. Deus EM Machina: On-Touch Contextual Functionality for Smart IoT Appliances. In Proceedings of CHI (Conference on Human Factors in Computing Systems). https://doi.org/10.1145/3025453.3025828

[23] Chouchang Yang and Alanson P Sample. 2016. EM-ID: Tag-less identification of electrical devices via electromagnetic emissions. In 2016 IEEE International Conference on RFID (RFID). IEEE, 1-8. 